\title{
Facile Syntheses of 2,2-Dimethyl-6-(2-oxoalkyl)-1,3-dioxin-4-ones and the Corresponding 6-Substituted 4-hydroxy-2-pyrones
}

\author{
Alan R. Katritzky, 'Zuoquan Wang, Mingyi Wang, C. Dennis Hall, and Kazuyuki Suzuki
}

Center for Heterocyclic Compounds, University of Florida, Department of Chemistry, Gainesville,

Florida 32611-7200

\section{katritzky@chem.ufl.edu}

Table of Contents

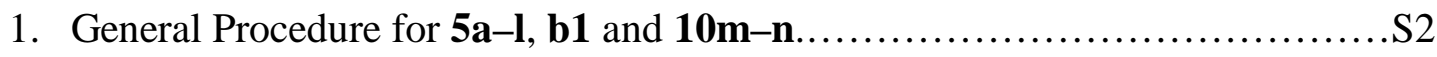

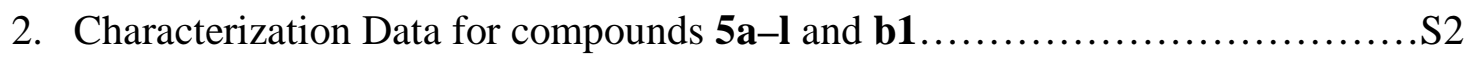

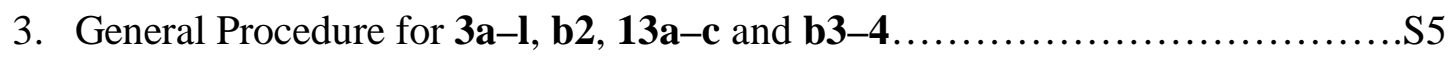

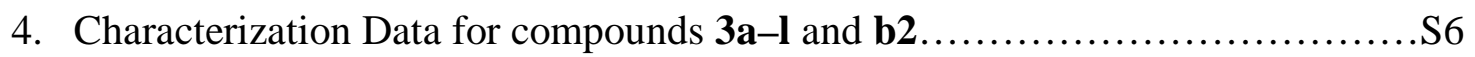

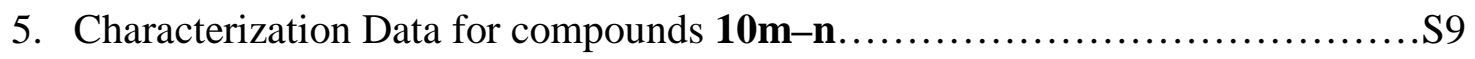

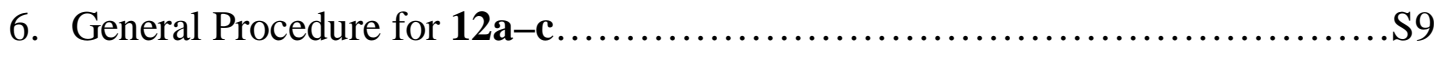

7. Characterization Data for compounds $\mathbf{1 2 a}-\mathbf{c}, \mathbf{1 3 a}-\mathbf{c}$ and $\mathbf{b 3}-\mathbf{4} \ldots \ldots \ldots \ldots \ldots \ldots . . . . . .6$ 


\section{General Procedure for the Preparation of 2,2-Dimethyl-6-(2-oxoalkyl)-1,3-dioxin-4-ones 5a-l,}

Byproduct b1 and $N, N$-Diisopropylacetamides 10m-n. To a solution of diisopropylamine $(99 \%, 0.77$

$\mathrm{g}, 7.5 \mathrm{mmol})$ in THF $(20 \mathrm{~mL})$ at $-78{ }^{\circ} \mathrm{C}$ was added $n$-BuLi $(1.6 \mathrm{M}, 5.16 \mathrm{~mL}, 8.25 \mathrm{mmol})$ dropwise over ca. $30 \mathrm{~min}$. A solution of 2,2,6-trimethyl-1,3-dioxin-4-one 4 (95\%, $0.85 \mathrm{~g}, 5.68 \mathrm{mmol})$ in THF (20 mL) was then added dropwise at $-78{ }^{\circ} \mathrm{C}$ for $10-15 \mathrm{~min}$. After stirring for $1.5 \mathrm{~h}$, a solution of 1acylbenzotriazole 9a-n $(5 \mathrm{mmol})$ in THF $(20 \mathrm{~mL})$ was added at $-78{ }^{\circ} \mathrm{C}$. The resulting mixture was stirred at $-78{ }^{\circ} \mathrm{C}$ and allowed to warm to room temperature overnight. Saturated aqueous ammonium chloride solution $(2 \mathrm{~mL})$ was added to quench the reaction, and the reaction mixture was concentrated under reduced pressure. Water $(100 \mathrm{~mL})$ was added to the residue, and the solution was extracted with ethyl acetate $(3 \times 50 \mathrm{~mL})$. The combined organic phase was washed with saturated aqueous sodium carbonate solution $(100 \mathrm{~mL})$, and dried over anhyd. $\mathrm{MgSO}_{4}$. After evaporation of the solvent, the residue was purified by column chromatography on silica gel to give 2,2-dimethyl-6-(2-oxoalkyl)-1,3dioxin-4-ones 5a-l, byproduct b1 and $N, N$-diisopropylacetamides 10m-n.

The ${ }^{1} \mathrm{H}$ NMR spectra of 5a-l show a new set of singlets at 3.32-4.04 ppm assigned to the methylene group attached to position 6 of the resultant 1,3-dioxin-4-one rings. The ${ }^{13} \mathrm{C}$ NMR spectra of 5a-l show characteristic carbonyl signals for ketones at 181.6 to $208.6 \mathrm{ppm}$ and the absence of signals for the benzotriazole group on 1-acylbenzotriazoles $\mathbf{9 a - 1 . ~}$

2,2-Dimethyl-6-(2-oxo-2-phenylethyl)-4H-1,3-dioxin-4-one $\quad(5 a): \quad$ colorless $\quad$ prisms after chromatography on silica gel using hexanes/ethyl acetate as eluant $(9 / 1, \mathrm{v} / \mathrm{v}) ; \mathrm{mp} 105-107{ }^{\circ} \mathrm{C}\left[\mathrm{lit}^{8}{ }^{8} \mathrm{mp}\right.$ 110.0-112.0 ${ }^{\circ} \mathrm{C}$; ; yield 65\%; ${ }^{1} \mathrm{H}$ NMR $\left(\mathrm{CDCl}_{3}\right) \delta 1.71(\mathrm{~s}, 6 \mathrm{H}), 3.91(\mathrm{~s}, 2 \mathrm{H}), 5.43(\mathrm{~s}, 1 \mathrm{H}), 7.46-7.54(\mathrm{~m}$, 2H), 7.60-7.66(m, 1H), 7.91-7.96 (m, 2H); ${ }^{13} \mathrm{C} \mathrm{NMR}\left(\mathrm{CDCl}_{3}\right) \delta 24.9(2 \mathrm{C}), 43.2,96.9,107.3,128.3$ (2C), 128.9 (2C), 134.0, 135.8, 160.7, 165.1, 193.0. Anal. Calcd for $\mathrm{C}_{14} \mathrm{H}_{14} \mathrm{O}_{4}$ : C, 68.28; H, 5.73. Found: C, 68.40; H, 5.80.

2,2-Dimethyl-6-[2-(4-methylphenyl)-2-oxoethyl]-4H-1,3-dioxin-4-one (5b): colorless needles after chromatography on silica gel using hexanes/chloroform as eluant $\left(6 / 1 \rightarrow 3 / 1\right.$, v/v); mp 87.0-88.0 ${ }^{\circ} \mathrm{C}$; 
yield 58\%; ${ }^{1} \mathrm{H}$ NMR $\left(\mathrm{CDCl}_{3}\right) \delta 1.70(\mathrm{~s}, 6 \mathrm{H}), 2.43(\mathrm{~s}, 3 \mathrm{H}), 3.88(\mathrm{~s}, 2 \mathrm{H}), 5.41(\mathrm{~s}, 1 \mathrm{H}), 7.30(\mathrm{~d}, J=8.2$ $\mathrm{Hz}, 2 \mathrm{H}), 7.83(\mathrm{~d}, J=8.2 \mathrm{~Hz}, 2 \mathrm{H}) ;{ }^{13} \mathrm{C} \mathrm{NMR}\left(\mathrm{CDCl}_{3}\right) \delta 21.6,24.9(2 \mathrm{C}), 43.1,96.7,107.1,128.3(2 \mathrm{C})$, 129.5 (2C), 133.3, 145.0, 160.7, 165.3, 192.6. Anal. Calcd for $\mathrm{C}_{15} \mathrm{H}_{16} \mathrm{O}_{4}: \mathrm{C}, 69.21 ; \mathrm{H}, 6.21$. Found: $\mathrm{C}$, 69.18; H, 6.30.

6-[2-(4-chlorophenyl)-2-oxoethyl]-2,2-dimethyl-4H-1,3-dioxin-4-one (5c): colorless needles after chromatography on silica gel using hexanes/chloroform as eluant $\left(6 / 1 \rightarrow 3 / 1\right.$, v/v); mp $117-119{ }^{\circ} \mathrm{C}$; yield $61 \% ;{ }^{1} \mathrm{H}$ NMR $\left(\mathrm{CDCl}_{3}\right) \delta 1.71(\mathrm{~s}, 6 \mathrm{H}), 5.42(\mathrm{~s}, 1 \mathrm{H}), 3.88(\mathrm{~s}, 2 \mathrm{H}), 7.48(\mathrm{~d}, J=8.5 \mathrm{~Hz}, 2 \mathrm{H}), 7.88$ $(\mathrm{d}, J=8.5 \mathrm{~Hz}, 2 \mathrm{H}) ;{ }^{13} \mathrm{C} \mathrm{NMR}\left(\mathrm{CDCl}_{3}\right) \delta 25.0(2 \mathrm{C}), 43.2,97.1,107.3,129.2(2 \mathrm{C}), 129.79(2 \mathrm{C}), 134.1$, 140.6, 160.6, 164.7, 191.8. Anal. Calcd for $\mathrm{C}_{14} \mathrm{H}_{13} \mathrm{ClO}_{4}$ : C, 59.90; H, 4.68. Found: C, 59.68; H, 4.57.

2,2-Dimethyl-6-(2-oxo-3,3-diphenylpropyl)-4H-1,3-dioxin-4-one (5d): colorless prisms after chromatography on silica gel using hexanes/ethyl acetate as eluant $(100 / 1 \rightarrow 6 / 1, \mathrm{v} / \mathrm{v})$; mp $87-89{ }^{\circ} \mathrm{C}$; yield 58\%; ${ }^{1} \mathrm{H}$ NMR $\left(\mathrm{CDCl}_{3}\right) \delta 1.68(\mathrm{~s}, 6 \mathrm{H}), 3.43(\mathrm{~s}, 2 \mathrm{H}), 5.16(\mathrm{~s}, 1 \mathrm{H}), 5.26(\mathrm{~s}, 1 \mathrm{H}), 7.18-7.24(\mathrm{~m}$, 4H), 7.28-7.39 (m, 6H); ${ }^{13} \mathrm{C} \mathrm{NMR}\left(\mathrm{CDCl}_{3}\right) \delta 25.0(2 \mathrm{C}), 46.5,64.5,96.7,107.2,127.7(2 \mathrm{C}), 128.9$ (8C), 37.0 (2C), 160.6, 164.4, 201.2. HRMS (EI) Calcd for $\mathrm{C}_{21} \mathrm{H}_{20} \mathrm{O}_{4}: m / z$ 336.1362. Found: 336.1353.

2,2-Dimethyl-6-[2-(2-naphthyl)-2-oxoethyl]-4H-1,3-dioxin-4-one (5e): yellowish prisms after chromatography on silica gel using hexanes/ethyl acetate as eluant $(20 / 1 \rightarrow 4 / 1, \mathrm{v} / \mathrm{v}) ; \mathrm{mp} 103-105{ }^{\circ} \mathrm{C}$; yield 65\%; ${ }^{1} \mathrm{H}$ NMR $\left(\mathrm{CDCl}_{3}\right) \delta 1.72(\mathrm{~s}, 6 \mathrm{H}), 4.04(\mathrm{~s}, 2 \mathrm{H}), 5.48(\mathrm{~s}, 1 \mathrm{H}), 7.56-7.67(\mathrm{~m}, 2 \mathrm{H}), 7.88-8.01$ $(\mathrm{m}, 4 \mathrm{H}), 8.45(\mathrm{~s}, 1 \mathrm{H}) ;{ }^{13} \mathrm{C} \mathrm{NMR}\left(\mathrm{CDCl}_{3}\right) \delta 25.0(2 \mathrm{C}), 43.3,97.0,107.3,123.5,127.2,127.8,128.9$, 129.1, 129.6, 130.4, 132.3, 133.1, 135.9, 160.8, 165.2, 192.9. Anal. Calcd for $\mathrm{C}_{18} \mathrm{H}_{16} \mathrm{O}_{4}: \mathrm{C}, 72.96 ; \mathrm{H}$, 5.44. Found: C, 72.84; H, 5.63.

2,2-Dimethyl-6-(2-oxooctyl)-4H-1,3-dioxin-4-one (5f): colorless oil after chromatography on silica gel using hexanes/ethyl acetate as eluant $(20 / 1 \rightarrow 5 / 1, \mathrm{v} / \mathrm{v})$; yield $53 \%$; ${ }^{1} \mathrm{H} \mathrm{NMR}\left(\mathrm{CDCl}_{3}\right) \delta 0.89(\mathrm{t}, J=$ $6.4 \mathrm{~Hz}, 3 \mathrm{H}), 1.22-1.35(\mathrm{~m}, 6 \mathrm{H}), 1.52-1.65(\mathrm{~m}, 2 \mathrm{H}), 1.72(\mathrm{~s}, 6 \mathrm{H}), 2.50(\mathrm{t}, J=7.3 \mathrm{~Hz}, 2 \mathrm{H}), 3.33(\mathrm{~s}, 2 \mathrm{H})$, 
$5.34(\mathrm{~s}, 1 \mathrm{H}) ;{ }^{13} \mathrm{C} \mathrm{NMR}\left(\mathrm{CDCl}_{3}\right) \delta 13.9,22.4,23.4,25.0(2 \mathrm{C}), 28.6,31.5,43.1,47.0,96.6,107.1$, 160.6, 164.6, 203.3. Anal. Calcd for $\mathrm{C}_{14} \mathrm{H}_{22} \mathrm{O}_{4}$ : C, 66.12; H, 8.72. Found: C, 66.28; H, 8.95.

6-(3,3-Dimethyl-2-oxobutyl)-2,2-dimethyl-4H-1,3-dioxin-4-one $\quad \mathbf{( 5 g ) :}$ colorless prisms after chromatography on silica gel using hexanes/ethyl acetate as eluant $(20 / 1 \rightarrow 6 / 1, \mathrm{v} / \mathrm{v}) ; \mathrm{mp} 76.0-78.0{ }^{\circ} \mathrm{C}$; yield 52\%; ${ }^{1} \mathrm{H}$ NMR $\left(\mathrm{CDCl}_{3}\right) \delta 1.19(\mathrm{~s}, 9 \mathrm{H}), 1.71(\mathrm{~s}, 6 \mathrm{H}), 3.43(\mathrm{~s}, 2 \mathrm{H}), 5.32(\mathrm{~s}, 1 \mathrm{H}) ;{ }^{13} \mathrm{C} \mathrm{NMR}\left(\mathrm{CDCl}_{3}\right)$ $\delta 24.9$ (2C), 26.0 (3C), 41.1, 44.8, 96.7, 107.1, 160.8, 165.6, 208.6. Anal. Calcd for $\mathrm{C}_{12} \mathrm{H}_{18} \mathrm{O}_{4}: \mathrm{C}_{\text {, }}$ 63.70; H, 8.02. Found: C, 63.87; H, 8.26.

2,2-Dimethyl-6-(3-methyl-2-oxobutyl)-4H-1,3-dioxin-4-one $\quad(5 \mathrm{~h}): \quad$ colorless $\quad$ oil after chromatography on silica gel using hexanes/ethyl acetate as eluant $\left(20 / 1 \rightarrow 6 / 1\right.$, v/v); yield $51 \%$; ${ }^{1} \mathrm{H}$ $\operatorname{NMR}\left(\mathrm{CDCl}_{3}\right) \delta 1.15(\mathrm{~d}, J=7.0 \mathrm{~Hz}, 6 \mathrm{H}), 1.71(\mathrm{~s}, 6 \mathrm{H}), 2.68($ septet, $J=7.0 \mathrm{~Hz}, 1 \mathrm{H}), 3.38(\mathrm{~s}, 2 \mathrm{H}), 5.33$ (s, 1H); ${ }^{13} \mathrm{C}$ NMR $\left(\mathrm{CDCl}_{3}\right) \delta 17.8$ (2C), 24.9 (2C), 41.3, 44.6, 96.6, 107.1, 160.7, 164.9, 207.0. Anal. Calcd for $\mathrm{C}_{11} \mathrm{H}_{16} \mathrm{O}_{4}$ : C, 62.25; H, 7.60. Found: C, 62.30; H, 7.82.

2,2-Dimethyl-6-(2-oxo-11-dodecenyl)-4H-1,3-dioxin-4-one (5i): yellow oil after chromatography on silica gel using hexanes/ethyl acetate as eluant $\left(20 / 1 \rightarrow 8 / 1\right.$, v/v); yield $51 \% ;{ }^{1} \mathrm{H} \mathrm{NMR}\left(\mathrm{CDCl}_{3}\right) \delta 1.28-$ $1.42(\mathrm{~m}, 10 \mathrm{H}), 1.52-1.65(\mathrm{~m}, 2 \mathrm{H}), 1.71(\mathrm{~s}, 6 \mathrm{H}), 2.04(\mathrm{q}, J=6.9 \mathrm{~Hz}, 2 \mathrm{H}), 2.50(\mathrm{t}, J=7.4 \mathrm{~Hz}, 2 \mathrm{H}), 3.32$ $(\mathrm{s}, 2 \mathrm{H}), 4.91-5.02(\mathrm{~m}, 2 \mathrm{H}), 5.34(\mathrm{~s}, 1 \mathrm{H}), 5.74-5.87(\mathrm{~m}, 1 \mathrm{H}) ;{ }^{13} \mathrm{C} \mathrm{NMR}\left(\mathrm{CDCl}_{3}\right) \delta 23.3,25.0(2 \mathrm{C}), 28.8$, 28.9, 29.0, 29.1, 29.2, 33.7, 43.1, 47.0, 96.6, 107.1, 114.1, 139.0, 160.7, 164.6, 203.4. Anal. Calcd for $\mathrm{C}_{18} \mathrm{H}_{28} \mathrm{O}_{4}$ : C, 70.10; H, 9.15. Found: C, 70.11; H, 9.52.

N,N-diisopropyl-3-phenyl-3-(2,2,6-trimethyl-4-oxo-4H-1,3-dioxin-5-yl)propanamide (b1): sticky oil after chromatography on silica gel using hexanes/ethyl acetate as eluant $(50 / 1 \rightarrow 3 / 1$, v/v, the second fraction); yield $21 \% ;{ }^{1} \mathrm{H} \mathrm{NMR}\left(\mathrm{CDCl}_{3}\right) \delta 1.11(\mathrm{~d}, J=6.7 \mathrm{~Hz}, 3 \mathrm{H}), 1.17(\mathrm{~d}, J=6.6 \mathrm{~Hz}, 3 \mathrm{H}), 1.34(\mathrm{~d}, J=$ $6.3 \mathrm{~Hz}, 3 \mathrm{H}), 1.36(\mathrm{~d}, J=5.8 \mathrm{~Hz}, 3 \mathrm{H}), 160(\mathrm{~s}, 3 \mathrm{H}), 1.68(\mathrm{~s}, 3 \mathrm{H}), 2.08(\mathrm{~s}, 3 \mathrm{H}), 2.76(\mathrm{dd}, J=15.7,5.0$ $\mathrm{Hz}, 1 \mathrm{H}), 3.34-3.53(\mathrm{~m}, 1 \mathrm{H}), 3.65(\mathrm{dd}, J=15.7,10.1 \mathrm{~Hz}, 1 \mathrm{H}), 4.08$ (septet, $J=6.7 \mathrm{~Hz}, 1 \mathrm{H}), 4.31(\mathrm{dd}, J$ 
$=10.1,5.0 \mathrm{~Hz}, 1 \mathrm{H}), 7.15-7.23(\mathrm{~m}, 1 \mathrm{H}), 7.24-7.32(\mathrm{~m}, 2 \mathrm{H}), 7.33-7.40(\mathrm{~m}, 2 \mathrm{H}) ;{ }^{13} \mathrm{C} \mathrm{NMR}\left(\mathrm{CDCl}_{3}\right) \delta$ 18.0, 20.7 (2C), 20.8 (2C), 23.9, 26.0, 38.4, 39.5, 45.6, 48.4, 104.7, 107.1, 126.3, 127.7 (2C), 128.4 (2C), 143.5, 161.7, 165.9, 170.4. Anal. Calcd for $\mathrm{C}_{22} \mathrm{H}_{31} \mathrm{NO}_{4}$ : C, 70.75; H, 8.37; N, 3.75. Found: C, $70.72 ; \mathrm{H}, 8.54 ; \mathrm{N}, 3.50$.

2,2-Dimethyl-6-[(E)-2-oxo-4-phenyl-3-butenyl]-4H-1,3-dioxin-4-one (5j): colorless prisms ${ }^{10 a}$ after chromatography on silica gel using hexanes/ethyl acetate as eluant $(50 / 1 \rightarrow 3 / 1$, v/v, the third fraction); mp 101-102 ${ }^{\circ} \mathrm{C}$; yield 37\%; ${ }^{1} \mathrm{H}$ NMR $\left(\mathrm{CDCl}_{3}\right) \delta 1.72(\mathrm{~s}, 6 \mathrm{H}), 3.60(\mathrm{~s}, 2 \mathrm{H}), 5.43(\mathrm{~s}, 1 \mathrm{H}), 6.77(\mathrm{~d}, J=$ $16.1 \mathrm{~Hz}, 1 \mathrm{H}), 7.38-7.46(\mathrm{~m}, 3 \mathrm{H}), 7.52-7.58(\mathrm{~m}, 2 \mathrm{H}), 7.62(\mathrm{~d}, J=16.1 \mathrm{~Hz}, 1 \mathrm{H}) ;{ }^{13} \mathrm{C} \mathrm{NMR}\left(\mathrm{CDCl}_{3}\right) \delta$ 24.9 (2C), 45.3, 96.6, 107.1, 124.6, 128.4 (2C), 129.0 (2C), 131.1, 133.7, 144.7, 160.6, 164.9, 192.3. Anal. Calcd for $\mathrm{C}_{16} \mathrm{H}_{16} \mathrm{O}_{4}$ : C, 70.58; H, 5.92. Found: C, 70.59; H, 5.99.

6-[2-(2-Furyl)-2-oxoethyl]-2,2-dimethyl-4H-1,3-dioxin-4-one $\quad \mathbf{( 5 k ) :}$ colorless prisms after chromatography on silica gel using hexanes/ethyl acetate as eluant $(6 / 1 \rightarrow 3 / 1, \mathrm{v} / \mathrm{v})$; mp $72-74{ }^{\circ} \mathrm{C}$; yield $66 \% ;{ }^{1} \mathrm{H} \mathrm{NMR}\left(\mathrm{CDCl}_{3}\right) \delta 1.70(\mathrm{~s}, 6 \mathrm{H}), 3.77(\mathrm{~s}, 2 \mathrm{H}), 5.44(\mathrm{~s}, 1 \mathrm{H}), 6.60(\mathrm{dd}, J=3.6,1.7 \mathrm{~Hz}, 1 \mathrm{H}), 7.28$ $(\mathrm{dd}, J=3.6,0.6 \mathrm{~Hz}, 1 \mathrm{H}), 7.64(\mathrm{dd}, J=1.7,0.6 \mathrm{~Hz}, 1 \mathrm{H}) ;{ }^{13} \mathrm{C} \mathrm{NMR}\left(\mathrm{CDCl}_{3}\right) \delta 24.8(2 \mathrm{C}), 42.9,96.8$, 107.3, 112.8, 118.4, 147.2, 151.6, 160.8, 164.5, 181.6. Anal. Calcd for $\mathrm{C}_{12} \mathrm{H}_{12} \mathrm{O}_{5}: \mathrm{C}, 61.02 ; \mathrm{H}, 5.13$. Found: C, 61.15; H, 5.06.

2,2-Dimethyl-6-[2-oxo-2-(2-thienyl)ethyl]-4H-1,3-dioxin-4-one $\quad$ (5I): yellow prisms after chromatography on silica gel using hexanes/chloroform as eluant $(5 / 1 \rightarrow 3 / 1, \mathrm{v} / \mathrm{v})$; mp $70-72{ }^{\circ} \mathrm{C}$; yield $60 \% ;{ }^{1} \mathrm{H}$ NMR $\left(\mathrm{CDCl}_{3}\right) \delta 1.71(\mathrm{~s}, 6 \mathrm{H}), 3.84(\mathrm{~s}, 2 \mathrm{H}), 5.45(\mathrm{~s}, 1 \mathrm{H}), 7.18(\mathrm{dd}, J=4.8,3.9 \mathrm{~Hz}, 1 \mathrm{H}), 7.72-$ $7.76(\mathrm{~m}, 2 \mathrm{H}) ;{ }^{13} \mathrm{C} \mathrm{NMR}\left(\mathrm{CDCl}_{3}\right) \delta 24.9(2 \mathrm{C}), 43.8,96.9,107.3,128.4,133.0,135.1,142.8,160.7$, 164.5, 185.5. Anal. Calcd for $\mathrm{C}_{12} \mathrm{H}_{12} \mathrm{O}_{4} \mathrm{~S}: \mathrm{C}, 57.12$; H, 4.80. Found: C, 56.95; H, 4.69.

General Procedure for the Preparation of 4-Hydroxy-6-substituted-2-pyrones 3a-l, Byproduct b2, 5-Alkyl-4-hydroxy-6-phenyl-2H-pyran-2-one 13a-c and Byproducts b3-4. A solution of each 
2,2-dimethyl-6-(2-oxoalkyl)-1,3-dioxin-4-one (5a-l) or 2,2-dimethyl-6-(1-alkyl-2-oxo-2-phenylethyl)1,3-dioxin-4-one (12a-c) $(3.0 \mathrm{mmol})$ in toluene $(30 \mathrm{~mL})$ was heated under reflux for $20-30 \mathrm{~min}$. The solvent was then removed under reduced pressure and the residue was purified by column chromatography on silica gel to give 4-hydroxy-6-substituted-2-pyrones 3a-l, byproduct b2, 5-alkyl-2pyrones 13a-c and byproducts $b 3-4$.

The ${ }^{1} \mathrm{H}$ NMR spectra of 3a-l show two sets of doublets for $\mathrm{H}^{3}$ at 5.27-5.59 ppm and $\mathrm{H}^{5}$ at 5.76-6.93 ppm, respectively, with typical meta coupling between $\mathrm{H}^{3}$ and $\mathrm{H}^{5}$ of 2-pyrone rings in the range 1.4-2.1 Hz. The ${ }^{13} \mathrm{C}$ NMR spectra of 3a-l display the existence of two ring methine carbons: C-3 at 88.8-90.0 ppm, C-5 at 96.5-102.1 ppm.

4-Hydroxy-6-phenyl-2H-pyran-2-one (3a): off-white microcrystals after chromatography on silica gel using hexanes/ethyl acetate as eluant $\left(20 / 1 \rightarrow 2 / 1\right.$, v/v); mp $250-251{ }^{\circ} \mathrm{C}\left[1\right.$ lit. $^{8} \mathrm{mp} 246{ }^{\circ} \mathrm{C}$ ]; yield 82\%; ${ }^{1} \mathrm{H}$ NMR (DMSO- $\left.d_{6}\right) \delta 5.41(\mathrm{~d}, J=1.8 \mathrm{~Hz}, 1 \mathrm{H}), 6.77(\mathrm{~d}, J=1.8 \mathrm{~Hz}, 1 \mathrm{H}), 7.47-7.56(\mathrm{~m}, 3 \mathrm{H})$, 7.81-7.90 (m, 2H), 11.87 (br s, 1H); ${ }^{13} \mathrm{C}$ NMR (DMSO- $\left.d_{6}\right) \delta 89.6,98.4,125.4(2 \mathrm{C}), 129.0(2 \mathrm{C}), 130.9$, $131.0,160.0,163.0,170.5$.

4-Hydroxy-6-(4-methylphenyl)-2H-pyran-2-one $\quad(3 \mathrm{~b}): \quad$ yellowish microcrystals after chromatography on silica gel using hexanes/ethyl acetate as eluant $(6 / 1 \rightarrow 1 / 1, \mathrm{v} / \mathrm{v}) ; \mathrm{mp} 206-208{ }^{\circ} \mathrm{C}$ $\left[\right.$ lit. ${ }^{19} \mathrm{mp} 212-213{ }^{\circ} \mathrm{C}$; ; yield $77 \% ;{ }^{1} \mathrm{H}$ NMR (DMSO- $\left.d_{6}\right) \delta 2.37(\mathrm{~s}, 3 \mathrm{H}), 5.37(\mathrm{~d}, J=2.0 \mathrm{~Hz}, 1 \mathrm{H}), 6.71$ $(\mathrm{d}, J=2.0 \mathrm{~Hz}, 1 \mathrm{H}), 7.33(\mathrm{~d}, J=8.2 \mathrm{~Hz}, 2 \mathrm{H}), 7.75(\mathrm{~d}, J=8.2 \mathrm{~Hz}, 2 \mathrm{H}), 11.83($ br s, $1 \mathrm{H}) ;{ }^{13} \mathrm{C} \mathrm{NMR}$ (DMSO- $\left.d_{6}\right) \delta 20.9,89.3,97.6,125.4$ (2C), 128.3, 129.6 (2C), 141.0, 160.2, 163.1, 170.6. Anal. Calcd for $\mathrm{C}_{12} \mathrm{H}_{10} \mathrm{O}_{3}: \mathrm{C}, 71.28 ; \mathrm{H}, 4.98$. Found: $\mathrm{C}, 71.03 ; \mathrm{H}, 5.01$.

6-(4-Chlorophenyl)-4-hydroxy-2H-pyran-2-one (3c): yellowish microcrystals after chromatography on silica gel using hexanes/ethyl acetate as eluant $\left(3 / 1 \rightarrow 1 / 1\right.$, v/v); mp $228-230{ }^{\circ} \mathrm{C}\left[\mathrm{lit}^{20}{ }^{20} \mathrm{mp} 250-252\right.$ ${ }^{\circ} \mathrm{C}$ for the monohydrate]; yield $75 \% ;{ }^{1} \mathrm{H}$ NMR (DMSO- $\left.d_{6}\right) \delta 5.41(\mathrm{~d}, J=1.8 \mathrm{~Hz}, 1 \mathrm{H}), 6.81(\mathrm{~d}, J=1.8$ 
$\mathrm{Hz}, 1 \mathrm{H}), 7.58(\mathrm{~d}, J=8.6 \mathrm{~Hz}, 2 \mathrm{H}), 7.88(\mathrm{~d}, J=8.6 \mathrm{~Hz}, 2 \mathrm{H}), 11.92(\mathrm{br} \mathrm{s}, 1 \mathrm{H}) ;{ }^{13} \mathrm{C} \mathrm{NMR}\left(\mathrm{DMSO}-d_{6}\right) \delta$ 89.9, 98.9, 127.3 (2C), 129.1 (2C), 129.9, 135.6, 158.8, 162.8, 170.5. Anal. Calcd for $\mathrm{C}_{11} \mathrm{H}_{7} \mathrm{ClO}_{3}$ : C, 59.35; H, 3.18. Found: C, 59.10; H, 3.23.

6-Benzhydryl-4-hydroxy-2H-pyran-2-one (3d): colorless microcrystals after chromatography on silica gel using hexanes/ethyl acetate as eluant $\left(6 / 1 \rightarrow 1 / 1\right.$, v/v); mp $186-187{ }^{\circ} \mathrm{C}$; yield $85 \%$; ${ }^{1} \mathrm{H}$ NMR $\left(\mathrm{DMSO}-d_{6}\right) \delta 5.27(\mathrm{~d}, J=1.9 \mathrm{~Hz}, 1 \mathrm{H}), 5.44(\mathrm{~s}, 1 \mathrm{H}), 5.76(\mathrm{~d}, J=1.9 \mathrm{~Hz}, 1 \mathrm{H}), 7.20-7.41(\mathrm{~m}, 10 \mathrm{H})$, 11.73 (br s, $1 \mathrm{H}) ;{ }^{13} \mathrm{C}$ NMR (DMSO- $\left.d_{6}\right) \delta 53.9,88.8,102.1,127.2$ (2C), 128.7 (4C), 128.8 (4C), 139.5 (2C), 163.5, 166.5, 170.0. Anal. Calcd for $\mathrm{C}_{18} \mathrm{H}_{14} \mathrm{O}_{3}$ : C, 77.68; H, 5.07. Found: C, 77.79; H, 5.10.

(Z)-4-Hydroxy-4-(2-naphthyl)-3-buten-2-one (b2): yellow prisms after chromatography on silica gel using hexanes/ethyl acetate as eluan $\left(50 / 1 \rightarrow 40 / 1\right.$, v/v, the first fraction); mp $72-74{ }^{\circ} \mathrm{C}\left[\right.$ lit. $^{21} \mathrm{mp}$ $78-79{ }^{\circ} \mathrm{C}$; ; yield 14\%; ${ }^{1} \mathrm{H}$ NMR $\left(\mathrm{CDCl}_{3}\right) \delta 2.15(\mathrm{~s}, 3 \mathrm{H}), 6.23(\mathrm{~s}, 1 \mathrm{H}), 7.41-7.51(\mathrm{~m}, 2 \mathrm{H}), 7.75-7.86$ $(\mathrm{m}, 4 \mathrm{H}), 8.34(\mathrm{~s}, 1 \mathrm{H}), 16.16(\mathrm{br} \mathrm{s}, 1 \mathrm{H}) ;{ }^{13} \mathrm{C} \mathrm{NMR}\left(\mathrm{CDCl}_{3}\right) \delta 25.9,97.0,123.1,126.7,127.7,128.0$ 128.1, 128.4, 129.2, 132.1, 132.7, 135.2, 183.1, 193.8. Anal. Calcd for $\mathrm{C}_{14} \mathrm{H}_{12} \mathrm{O}_{2}$ : C, 79.22; 5.70. Found: C, 78.94; 5.85.

4-Hydroxy-6-(2-naphthyl)-2H-pyran-2-one (3e): off-white microcrystals after chromatography on silica gel using hexanes/ethyl acetate as eluant $\left(10 / 1 \rightarrow 1 / 1, \mathrm{v} / \mathrm{v}\right.$, the last fraction); mp $235-236{ }^{\circ} \mathrm{C}$; yield $66 \% ;{ }^{1} \mathrm{H}$ NMR (DMSO-d $)_{6} \delta 5.46(\mathrm{~d}, J=1.7 \mathrm{~Hz}, 1 \mathrm{H}), 6.93(\mathrm{~d}, J=1.7 \mathrm{~Hz}, 1 \mathrm{H}), 7.58-7.68(\mathrm{~m}, 2 \mathrm{H})$, 7.94-8.12 (m, 4H), $8.48(\mathrm{~s}, 1 \mathrm{H}), 11.97$ (br s, 1H); ${ }^{13} \mathrm{C}$ NMR (DMSO-d 6 ) $\delta 89.8,98.9,122.3,125.5$, 127.0, 127.6, 127.8, 128.3, 128.7, 129.0, 132.6, 133.8, 159.9, 163.1, 170.6. Anal. Calcd for $\mathrm{C}_{15} \mathrm{H}_{10} \mathrm{O}_{3}$ : C, 75.62; H, 4.23. Found: C, 75.38; H, 4.16.

6-Hexyl-4-hydroxy-2H-pyran-2-one (3f): yellowish microcrystals after chromatography on silica gel using hexanes/ethyl acetate as eluant $(20 / 1 \rightarrow 2 / 1, \mathrm{v} / \mathrm{v}) ;$ mp $56-57{ }^{\circ} \mathrm{C}$; yield $70 \% ;{ }^{1} \mathrm{H}$ NMR $\left(\mathrm{CDCl}_{3}\right)$ $\delta 0.88(\mathrm{t}, J=6.9 \mathrm{~Hz}, 3 \mathrm{H}), 1.20-1.40(\mathrm{~m}, 6 \mathrm{H}), 1.58-1.70(\mathrm{~m}, 2 \mathrm{H}), 2.48(\mathrm{t}, J=7.6 \mathrm{~Hz}, 2 \mathrm{H}), 5.57(\mathrm{~d}, J=$ 
$1.9 \mathrm{~Hz}, 1 \mathrm{H}), 5.97(\mathrm{~d}, J=1.9 \mathrm{~Hz}, 1 \mathrm{H}), 10.7(\mathrm{br} \mathrm{s}, 1 \mathrm{H}) ;{ }^{13} \mathrm{C} \mathrm{NMR}\left(\mathrm{CDCl}_{3}\right) \delta 14.0,22.4,26.6,28.6,31.4$, 33.6, 89.8, 101.3, 167.4, 168.4, 172.6. Anal. Calcd for $\mathrm{C}_{11} \mathrm{H}_{16} \mathrm{O}_{3}$ : C, 67.32; H, 8.22. Found: C, 67.22; H, 8.23.

6-(tert-Butyl)-4-hydroxy-2H-pyran-2-one (3g): yellowish prisms after chromatography on silica gel using hexanes/ethyl acetate as eluant $(20 / 1 \rightarrow 2 / 1, \mathrm{v} / \mathrm{v}) ; \mathrm{mp} 131-133{ }^{\circ} \mathrm{C}$; yield $86 \% ;{ }^{1} \mathrm{H} \mathrm{NMR}\left(\mathrm{CDCl}_{3}\right) \delta$ $1.26(\mathrm{~s}, 9 \mathrm{H}), 5.59(\mathrm{~d}, J=1.8 \mathrm{~Hz}, 1 \mathrm{H}), 6.06(\mathrm{~d}, J=1.8 \mathrm{~Hz}, 1 \mathrm{H}), 11.13(\mathrm{br} \mathrm{s}, 1 \mathrm{H}) ;{ }^{13} \mathrm{C} \mathrm{NMR}\left(\mathrm{CDCl}_{3}\right) \delta$ 27.7 (3C), 36.1, 89.7, 98.3, 168.2, 172,8, 174.0. Anal. Calcd for $\mathrm{C}_{9} \mathrm{H}_{12} \mathrm{O}_{3}$ : C, 64.27; H, 7.19. Found: C, 64.29; H, 7.26.

4-Hydroxy-6-isopropyl-2H-pyran-2-one (3h): yellowish microcrystals after chromatography on silica gel using hexanes/ethyl acetate as eluant $\left(20 / 1 \rightarrow 2 / 1\right.$, v/v); mp 82-84 ${ }^{\circ} \mathrm{C}$; yield $53 \%$; ${ }^{1} \mathrm{H}$ NMR $\left(\mathrm{CDCl}_{3}\right) \delta 1.23(\mathrm{~d}, J=6.9 \mathrm{~Hz}, 6 \mathrm{H}), 2.74($ septet, $J=6.9 \mathrm{~Hz}, 1 \mathrm{H}), 5.59(\mathrm{~d}, J=2.0 \mathrm{~Hz}, 1 \mathrm{H}), 5.99(\mathrm{~d}, J=$ $2.0 \mathrm{~Hz}, 1 \mathrm{H}), 10.95$ (br s, $1 \mathrm{H}) ;{ }^{13} \mathrm{C} \mathrm{NMR}\left(\mathrm{CDCl}_{3}\right) \delta 19.9$ (2C), 32.6, 89.8, 99.2, 168.3, 171.8, 172.8 . Anal. Calcd for $\mathrm{C}_{8} \mathrm{H}_{10} \mathrm{O}_{3}$ : C, 62.33; H, 6.54. Found: C, 62.45; H, 6.56.

6-(9-Decenyl)-4-hydroxy-2H-pyran-2-one (3i): yellowish prisms after chromatography on silica gel using hexanes/ethyl acetate as eluant $\left(5 / 1 \rightarrow 2 / 1\right.$, v/v); mp $62-64{ }^{\circ} \mathrm{C}$; yield $64 \%$; ${ }^{1} \mathrm{H}$ NMR $\left(\mathrm{CDCl}_{3}\right) \delta$ $1.22-1.43(\mathrm{~m}, 10 \mathrm{H}), 1.63$ (br quintet, $J=6.9 \mathrm{~Hz}, 2 \mathrm{H}), 2.03(\mathrm{q}, J=7.0 \mathrm{~Hz}, 2 \mathrm{H}), 2.48(\mathrm{t}, J=7.6 \mathrm{~Hz}$, 2H), 4.91-5.02 (m, 2H), $5.58(\mathrm{~d}, J=1.7 \mathrm{~Hz}, 1 \mathrm{H}), 5.73-5.88(\mathrm{~m}, 1 \mathrm{H}), 5.98(\mathrm{~d}, J=1.7 \mathrm{~Hz}, 1 \mathrm{H}), 11.28$ (br s, $1 \mathrm{H}) ;{ }^{13} \mathrm{C} \mathrm{NMR}\left(\mathrm{CDCl}_{3}\right) \delta 26.6,28.8,28.9,29.0,29.1,29.2,33.6,33.7,89.7,101.3,114.2,139.1$, 167.4, 168.4, 172.7. Anal. Calcd for $\mathrm{C}_{15} \mathrm{H}_{22} \mathrm{O}_{3}$ : C, 71.97; H, 8.86. Found: C, 71.89; H, 9.17.

\section{4-Hydroxy-6-[(E)-2-phenylethenyl]-2H-pyran-2-one $\quad(\mathbf{3 j}): \quad y^{2}$ ellowish $\quad$ prisms $^{10 a}$ after} chromatography on silica gel using hexanes/ethyl acetate as eluant $(10 / 1 \rightarrow 1 / 2, \mathrm{v} / \mathrm{v})$; mp $234-236{ }^{\circ} \mathrm{C}$; yield 73\%; ${ }^{1} \mathrm{H}$ NMR (DMSO- $\left.d_{6}\right) \delta 5.35(\mathrm{~d}, J=1.5 \mathrm{~Hz}, 1 \mathrm{H}), 6.25(\mathrm{~d}, J=1.5 \mathrm{~Hz}, 1 \mathrm{H}), 7.05(\mathrm{~d}, J=16.1$ $\mathrm{Hz}, 1 \mathrm{H}), 7.31(\mathrm{~d}, J=16.1 \mathrm{~Hz}, 1 \mathrm{H}), 7.35-7.46(\mathrm{~m}, 3 \mathrm{H}), 7.78(\mathrm{~d}, \mathrm{~J}=7.3 \mathrm{~Hz}, 2 \mathrm{H}), 11.8(\mathrm{~s}, 1 \mathrm{H}) ;{ }^{13} \mathrm{C} \mathrm{NMR}$ 
$\left(\mathrm{DMSO}-d_{6}\right) \delta 90.0,101.9,119.9,127.5,128.8,129.3,133.9,135.2,159.0,162.8,170.1$. Anal. Calcd for $\mathrm{C}_{13} \mathrm{H}_{10} \mathrm{O}_{3}: \mathrm{C}, 72.89 ; \mathrm{H}, 4.71$. Found: $\mathrm{C}, 72.71 ; \mathrm{H}, 4.94$.

6-(2-Furyl)-4-hydroxy-2H-pyran-2-one (3k): yellowish microcrystals ${ }^{10 a}$ after chromatography on silica gel using hexanes/ethyl acetate as eluant $(6 / 1 \rightarrow 1 / 1, \mathrm{v} / \mathrm{v}) ; \mathrm{mp} 202-204{ }^{\circ} \mathrm{C}$; yield $61 \%$; ${ }^{1} \mathrm{H}$ NMR $\left(\mathrm{DMSO}-d_{6}\right) \delta 5.27(\mathrm{~d}, J=2.1 \mathrm{~Hz}, 1 \mathrm{H}), 6.39(\mathrm{~d}, J=2.1 \mathrm{~Hz}, 1 \mathrm{H}), 6.71(\mathrm{dd}, J=3.4,1.4 \mathrm{~Hz}, 1 \mathrm{H}), 7.08(\mathrm{~d}$, $J=3.4 \mathrm{~Hz}, 1 \mathrm{H}), 7.92(\mathrm{~d}, J=1.4 \mathrm{~Hz}, 1 \mathrm{H}), 11.40($ br s, $1 \mathrm{H}) ;{ }^{13} \mathrm{C}$ NMR (DMSO- $\left.d_{6}\right) \delta 89.1,96.5,111.9$, 112.7, 145.7, 146.0, 152.1, 162.4, 170.8. HRMS (EI) Calcd for $\mathrm{C}_{9} \mathrm{H}_{6} \mathrm{O}_{4}: \mathrm{m} / z$ 178.0266. Found: 178.0261.

4-Hydroxy-6-(2-thienyl)-2H-pyran-2-one (3l): yellowish microcrystals after chromatography on silica gel using hexanes/ethyl acetate as eluant $(6 / 1 \rightarrow 1 / 1, \mathrm{v} / \mathrm{v}) ; \mathrm{mp} 214-216{ }^{\circ} \mathrm{C}$; yield $82 \%$; ${ }^{1} \mathrm{H}$ NMR $\left(\mathrm{DMSO}-d_{6}\right) \delta 5.33(\mathrm{~d}, J=1.4 \mathrm{~Hz}, 1 \mathrm{H}), 6.64(\mathrm{~d}, J=1.4 \mathrm{~Hz}, 1 \mathrm{H}), 7.22(\mathrm{dd}, J=4.8,3.7 \mathrm{~Hz}, 1 \mathrm{H}), 7.78(\mathrm{~d}$, $J=3.7 \mathrm{~Hz}, 1 \mathrm{H}), 7.83(\mathrm{~d}, J=4.8 \mathrm{~Hz}, 1 \mathrm{H}), 11.88(\mathrm{br} \mathrm{s}, 1 \mathrm{H}) ;{ }^{13} \mathrm{C}$ NMR (DMSO-d 6$) \delta 89.0,96.8,127.7$, 128.8, 130.2, 134.5, 156.1, 162.4, 170.5. Anal. Calcd for $\mathrm{C}_{9} \mathrm{H}_{6} \mathrm{O}_{3} \mathrm{~S}: \mathrm{C}, 55.66 ; \mathrm{H}, 3.11$. Found: C, 55.72; H, 3.05.

2-[1,1'-Biphenyl]-4-yl-N,N-diisopropylacetamide (10m): colorless prisms after chromatography on silica gel using hexanes/ethyl acetate as eluant $\left(20 / 1 \rightarrow 8 / 1\right.$, v/v); mp $116-118{ }^{\circ} \mathrm{C}$; yield $37 \%$; ${ }^{1} \mathrm{H}$ NMR $\left(\mathrm{CDCl}_{3}\right) \delta 1.05(\mathrm{~d}, J=6.6 \mathrm{~Hz}, 6 \mathrm{H}), 1.43(\mathrm{~d}, J=6.7 \mathrm{~Hz}, 6 \mathrm{H}), 3.40$ (br septet, $\left.1 \mathrm{H}\right), 3.72(\mathrm{~s}, 2 \mathrm{H}), 4.00$ (septet, $J=6.7 \mathrm{~Hz}, 1 \mathrm{H}), 7.28-7.36(\mathrm{~m}, 3 \mathrm{H}), 7.38-7.47(\mathrm{~m}, 2 \mathrm{H}), 7.52-7.62(\mathrm{~m}, 4 \mathrm{H}) ;{ }^{13} \mathrm{C} \mathrm{NMR}\left(\mathrm{CDCl}_{3}\right)$ $\delta 20.5$ (2C), 20.7 (2C), 42.9, 45.8, 49.4, 127.0 (2C), 127.1, 127.3 (2C), 128.7 (2C), 129.0 (2C), 134.9, 139.4, 140.9, 169.8. Anal. Calcd for $\mathrm{C}_{20} \mathrm{H}_{25} \mathrm{NO}$ : C, 81.31; H, 8.53; N, 4.74. Found: C, 81.09; H, 8.61; N, 4.38.

$N, N$-Diisopropyl-2-(phenylsulfanyl)acetamide (10n): yellow oil ${ }^{22}$ after chromatography on silica gel using hexanes/ethyl acetate as eluant $(20 / 1 \rightarrow 8 / 1, \mathrm{v} / \mathrm{v})$; yield $41 \% ;{ }^{1} \mathrm{H} \mathrm{NMR}\left(\mathrm{CDCl}_{3}\right) \delta 1.22(\mathrm{~d}, J=$ 
$6.6 \mathrm{~Hz}, 6 \mathrm{H}), 1.37(\mathrm{~d}, J=6.7 \mathrm{~Hz}, 6 \mathrm{H}), 3.41$ (br septet, $J=6.6 \mathrm{~Hz}, 1 \mathrm{H}), 3.74(\mathrm{~s}, 2 \mathrm{H}), 3.99$ (septet, $J=$

$6.7 \mathrm{~Hz}, 1 \mathrm{H}), 7.17-7.24(\mathrm{~m}, 1 \mathrm{H}), 7.25-7.33(\mathrm{~m}, 2 \mathrm{H}), 7.41-7.48(\mathrm{~m}, 2 \mathrm{H}) ;{ }^{13} \mathrm{C} \mathrm{NMR}\left(\mathrm{CDCl}_{3}\right) \delta 20.3$ (2C), 20.8 (2C), 39.2, 46.1, 49.8, 126.6, 128.9 (2C), 130.0 (2C), 135.5, 166.9. Anal. Calcd for $\mathrm{C}_{14} \mathrm{H}_{21} \mathrm{NOS}$ : C, 66.89; H, 8.42; N, 5.57. Found: C, 66.74; H, 8.51; 5.77.

\section{General Procedure for the Preparation of 2,2-Dimethyl-6-(1-alkyl-2-oxo-2-phenylethyl)-1,3-}

dioxin-4-one 12a-c. Sodium hydride $(60 \%, 3.25 \mathrm{mmol})$ was added to a stirred solution of 2,2dimethyl-6-(2-oxo-2-phenylethyl)-1,3-dioxin-4-one $5 \mathbf{a}(2.36 \mathrm{mmol})$ in $\mathrm{THF}(20 \mathrm{~mL})$ at room temperature and stirring was continued for $40 \mathrm{~min}$. A solution of alkyl iodide (EtI for 12a, $\mathrm{n}$-BuI for 12b, $\mathrm{n}-\mathrm{C}_{5} \mathrm{H}_{11} \mathrm{I}$ for $\left.12 \mathbf{c}, 2.38 \mathrm{mmol}\right)$ in THF $(20 \mathrm{~mL})$ was then added dropwise and the mixture was stirred for 15 days at room temperature. The solvent was removed under reduced pressure and the residue was purified by column chromatography on silica gel using a mixture of hexanes and ethyl acetate as eluant $(100 / 1 \rightarrow 6 / 1, v / v)$ to give dioxinones $\mathbf{1 2 a - c}$.

Analysis of 12a-c by ${ }^{1} \mathrm{H}$ NMR revealed the presence of two triplets at $0.87-1.01$ and $4.07-4.17 \mathrm{ppm}$, corresponding to new methyl and methine groups respectively. The two multiplets of each of $\mathbf{1 2 a}-\mathbf{c}$ at 1.77-1.96 and 1.97-2.17 ppm are due to the two protons of the methylene group, which are not magnetically equivalent as they attach to the carbon adjacent to a new chiral center. The ${ }^{1} \mathrm{H}$ NMR of 13a-c show singlets for $\mathrm{H}^{3}$ at 5.48-5.49 ppm, appearance of alkyl groups and absence of $\mathrm{H}^{5}$ as compared with 3a-l. Interestingly, all hydrogens in the phenyl groups of 13a-c resonate as apparent singlets at 7.51-7.52 ppm. The ${ }^{13} \mathrm{C}$ NMR of 13a-c display C-3 at $90.0 \mathrm{ppm}$, but C-5 at $112.5-113.6$ ppm, downfield from that of unsubstituted 3a-l at 96.5-102.1 ppm.

6-(1-Benzoylpropyl)-2,2-dimethyl-4H-1,3-dioxin-4-one (12a): colorless needles after chromatography on silica gel using hexanes/ethyl acetate as eluant $\left(100 / 1 \rightarrow 6 / 1\right.$, v/v); mp $73-74{ }^{\circ} \mathrm{C}$; yield $62 \% ;{ }^{1} \mathrm{H}$ NMR $\left(\mathrm{CDCl}_{3}\right) \delta 1.01(\mathrm{t}, J=7.4 \mathrm{~Hz}, 3 \mathrm{H}), 1.53(\mathrm{~s}, 3 \mathrm{H}), 1.58(\mathrm{~s}, 3 \mathrm{H}), 1.80-1.96(\mathrm{~m}, 1 \mathrm{H})$, 2.01-2.17 (m, 1H), $4.07(\mathrm{t}, J=7.1 \mathrm{~Hz}, 1 \mathrm{H}), 5.42(\mathrm{~s}, 1 \mathrm{H}), 7.45-7.53(\mathrm{~m}, 2 \mathrm{H}), 7.57-7.64(\mathrm{~m}, 1 \mathrm{H}), 7.91-$ $7.98(\mathrm{~m}, 2 \mathrm{H}) ;{ }^{13} \mathrm{C} \mathrm{NMR}\left(\mathrm{CDCl}_{3}\right) \delta 12.0,22.6,24.7,24.8,53.1,95.3,106.8,128.4(2 \mathrm{C}), 128.8(2 \mathrm{C})$, 
133.7, 136.3, 160.7, 168.4, 195.6. Anal. Calcd for $\mathrm{C}_{16} \mathrm{H}_{18} \mathrm{O}_{4}$ : C, 70.06; H, 6.61. Found: $\mathrm{C}, 70.15 ; \mathrm{H}$, 6.79 .

6-(1-Benzoylpentyl)-2,2-dimethyl-4H-1,3-dioxin-4-one (12b): yellow oil after chromatography on silica gel using hexanes/ethyl acetate as eluant $\left(10 / 1 \rightarrow 4 / 1\right.$, v/v); yield $52 \% ;{ }^{1} \mathrm{H}$ NMR $\left(\mathrm{CDCl}_{3}\right) \delta 0.90(\mathrm{t}$, $J=7.2 \mathrm{~Hz}, 3 \mathrm{H}), 1.25-1.42(\mathrm{~m}, 4 \mathrm{H}), 1.54(\mathrm{~s}, 3 \mathrm{H}), 1.58(\mathrm{~s}, 3 \mathrm{H}), 1.77-1.90(\mathrm{~m}, 1 \mathrm{H}), 1.97-2.09(\mathrm{~m}, 1 \mathrm{H})$, $4.15(\mathrm{t}, J=7.2 \mathrm{~Hz}, 1 \mathrm{H}), 5.41(\mathrm{~s}, 1 \mathrm{H}), 7.46-7.52(\mathrm{~m}, 2 \mathrm{H}), 7.58-7.64(\mathrm{~m}, 1 \mathrm{H}), 7.92-7.96(\mathrm{~m}, 2 \mathrm{H}) ;{ }^{13} \mathrm{C}$ $\operatorname{NMR}\left(\mathrm{CDCl}_{3}\right) \delta 13.8,22.5,24.6,24.8,28.9,29.5,51.4,95.1,106.8,128.4(2 \mathrm{C}), 128.8(2 \mathrm{C}), 133.7$, 136.1, 160.8, 168.6, 195.6. Anal. Calcd for $\mathrm{C}_{18} \mathrm{H}_{22} \mathrm{O}_{4}$ : C, 71.50; H, 7.33. Found: C, 71.79; H, 7.56.

6-(1-Benzoylhexyl)-2,2-dimethyl-4H-1,3-dioxin-4-one (12c): yellow oil after chromatography on silica gel using hexanes/ethyl acetate as eluant $(10 / 1 \rightarrow 4 / 1, \mathrm{v} / \mathrm{v})$; yield $51 \% ;{ }^{1} \mathrm{H}$ NMR $\left(\mathrm{CDCl}_{3}\right) \delta 0.87(\mathrm{t}$, $J=6.3 \mathrm{~Hz}, 3 \mathrm{H}), 1.23-1.42(\mathrm{~m}, 6 \mathrm{H}), 1.54(\mathrm{~s}, 3 \mathrm{H}), 1.58(\mathrm{~s}, 3 \mathrm{H}), 1.77-1.89(\mathrm{~m}, 1 \mathrm{H}), 1.97-2.09(\mathrm{~m}, 1 \mathrm{H})$, $4.17(\mathrm{t}, J=7.2 \mathrm{~Hz}, 1 \mathrm{H}), 5.42(\mathrm{~s}, 1 \mathrm{H}), 7.46-7.52(\mathrm{~m}, 2 \mathrm{H}), 7.58-7.64(\mathrm{~m}, 1 \mathrm{H}), 7.93-7.96(\mathrm{~m}, 2 \mathrm{H}),{ }^{13} \mathrm{C}$ NMR $\left(\mathrm{CDCl}_{3}\right) \delta 13.8,22.2,24.6,24.7,27.0,29.1,31.4,51.4,95.0,106.8,128.3(2 \mathrm{C}), 128.7(2 \mathrm{C})$, 133.7, 136.1, 160.7, 168.6, 195.6. Anal. Calcd for $\mathrm{C}_{19} \mathrm{H}_{24} \mathrm{O}_{4}$ : C, 72.13; H, 7.65. Found: $\mathrm{C}, 72.42 ; \mathrm{H}$, 7.96

5-Ethyl-4-hydroxy-6-phenyl-2H-pyran-2-one (13a): off-white microcrystals after chromatography on silica gel using hexanes/ethyl acetate as eluant $\left(20 / 1 \rightarrow 2 / 1\right.$, v/v); mp $212-214{ }^{\circ} \mathrm{C}$; yield $74 \% ;{ }^{1} \mathrm{H}$ NMR (DMSO- $\left.d_{6}\right) \delta 1.08(\mathrm{t}, J=7.3 \mathrm{~Hz}, 3 \mathrm{H}), 2.31(\mathrm{q}, J=7.3 \mathrm{~Hz}, 2 \mathrm{H}), 5.48(\mathrm{~s}, 1 \mathrm{H}), 7.52(\mathrm{~s}, 5 \mathrm{H}), 12.05$ (br s, $1 \mathrm{H}$ ); ${ }^{13} \mathrm{C}$ NMR (DMSO- $\left.d_{6}\right) \delta 14.2,18.2,90.0,113.6,128.3$ (2C), $128.6(2 \mathrm{C}), 129.9,132.6$, 158.0, 162.7, 170.3. Anal. Calcd for $\mathrm{C}_{13} \mathrm{H}_{12} \mathrm{O}_{3}: \mathrm{C}, 72.21 ; \mathrm{H}$, 5.59. Found: $\mathrm{C}, 71.89 ; \mathrm{H}, 5.58$.

2-Butyl-1-phenyl-1,3-butanedione (b3): colorless oil $^{23}$ after chromatography on silica gel using hexanes/ethyl acetate as eluant $\left(50 / 1 \rightarrow 20 / 1, \mathrm{v} / \mathrm{v}\right.$, the first fraction); yield $15 \% ;{ }^{1} \mathrm{H}$ NMR $\left(\mathrm{CDCl}_{3}\right) \delta 0.89$ (t, $J=7.1 \mathrm{~Hz}, 3 \mathrm{H}), 1.22-1.41(\mathrm{~m}, 4 \mathrm{H}), 1.89-2.10(\mathrm{~m}, 2 \mathrm{H}), 2.15(\mathrm{~s}, 3 \mathrm{H}), 4.43(\mathrm{t}, J=7.1 \mathrm{~Hz}, 1 \mathrm{H}), 7.47-$ 
$7.52(\mathrm{~m}, 2 \mathrm{H}), 7.58-7.63(\mathrm{~m}, 1 \mathrm{H}), 7.98-8.01(\mathrm{~m}, 2 \mathrm{H}) ;{ }^{13} \mathrm{C} \mathrm{NMR}\left(\mathrm{CDCl}_{3}\right) \delta 13.8,22.6,27.8,28.8,29.8$,

63.5, 128.6 (2C), 128.8 (2C), 133.7, 136.4, 196.4, 204.6. Anal. Calcd for $\mathrm{C}_{14} \mathrm{H}_{18} \mathrm{O}_{2}$ : C, 77.03; H, 8.31. Found: C, 77.38; H, 8.65.

5-Butyl-4-hydroxy-6-phenyl-2H-pyran-2-one (13b): colorless microcrystals after chromatography on silica gel using hexanes/ethyl acetate as eluant $\left(8 / 1 \rightarrow 1 / 1\right.$, v/v, the last fraction); mp $169-170{ }^{\circ} \mathrm{C}$; yield 26\%; ${ }^{1} \mathrm{H}$ NMR (DMSO- $\left.d_{6}\right) \delta 0.77(\mathrm{t}, J=7.3 \mathrm{~Hz}, 3 \mathrm{H}), 1.20($ sextet, $J=7.4 \mathrm{~Hz}, 2 \mathrm{H}), 1.39-1.50$ (m, 2H), 2.27-2.33 (m, 2H), $5.49(\mathrm{~s}, 1 \mathrm{H}), 7.52(\mathrm{~s}, 5 \mathrm{H}), 12.08($ br s, $1 \mathrm{H}) ;{ }^{13} \mathrm{C}$ NMR (DMSO- $\left.d_{6}\right) \delta 13.6$, 22.0, 24.3, 31.2, 90.0, 112.5, 128.4 (2C), 128.6 (2C), 129.9, 132.7, 158.2, 162.8, 170.4. Anal. Calcd for $\mathrm{C}_{15} \mathrm{H}_{16} \mathrm{O}_{3}$ : C, 73.75; H, 6.60. Found: C, 73.50; H, 6.90

2-Pentyl-1-phenyl-1,3-butanedione (b4): colorless oil ${ }^{24}$ after chromatography on silica gel using hexanes/ethyl acetate as eluant $\left(50 / 1 \rightarrow 30 / 1, \mathrm{v} / \mathrm{v}\right.$, the first fraction); yield $22 \% ;{ }^{1} \mathrm{H} \mathrm{NMR}\left(\mathrm{CDCl}_{3}\right) \delta 0.86$ $(\mathrm{t}, J=6.3 \mathrm{~Hz}, 3 \mathrm{H}), 1.20-1.42(\mathrm{~m}, 6 \mathrm{H}), 1.88-2.10(\mathrm{~m}, 2 \mathrm{H}), 2.15(\mathrm{~s}, 3 \mathrm{H}), 4.44(\mathrm{t}, J=7.1 \mathrm{~Hz}, 1 \mathrm{H}), 7.47-$ $7.52(\mathrm{~m}, 2 \mathrm{H}), 7.58-7.63(\mathrm{~m}, 1 \mathrm{H}), 7.98-8.01(\mathrm{~m}, 2 \mathrm{H}) ;{ }^{13} \mathrm{C} \mathrm{NMR}\left(\mathrm{CDCl}_{3}\right) \delta 13.9,22.3,27.4,27.8,29.0$ 31.6, 63.6, 128.6 (2C), 128.8 (2C), 133.6, 136.4, 196.4, 204.6. Anal. Calcd for $\mathrm{C}_{15} \mathrm{H}_{20} \mathrm{O}_{2}$ : C, 77.55; H, 8.68. Found: C, 77.58; H, 9.06.

4-Hydroxy-5-pentyl-6-phenyl-2H-pyran-2-one (13c): colorless microcrystals after chromatography on silica gel using hexanes/ethyl acetate as eluant $\left(8 / 1 \rightarrow 1 / 1, \mathrm{v} / \mathrm{v}\right.$, the last fraction); mp $160-162{ }^{\circ} \mathrm{C}$; yield $27 \%$; ${ }^{1} \mathrm{H}$ NMR (DMSO- $\left.d_{6}\right) \delta 0.79(\mathrm{t}, J=6.6 \mathrm{~Hz}, 3 \mathrm{H}), 1.09-1.25(\mathrm{~m}, 4 \mathrm{H}), 1.39-1.53(\mathrm{~m}, 2 \mathrm{H})$, 2.24-2.34 (m, 2H), $5.49(\mathrm{~s}, 1 \mathrm{H}), 7.51(\mathrm{~s}, 5 \mathrm{H}), 12.06($ br s, $1 \mathrm{H}) ;{ }^{13} \mathrm{C}$ NMR (DMSO-d $) \delta 13.8,21.6$, 24.4, 28.6, 31.0, 90.0, 112.5, 128.4 (2C), 128.6 (2C), 129.9, 132.7, 158.3, 162.8, 170.4. Anal. Calcd for $\mathrm{C}_{16} \mathrm{H}_{18} \mathrm{O}_{3}: \mathrm{C}, 74.39 ; \mathrm{H}, 7.04$. Found: C, 74.00; H, 7.45. 